\title{
"Maltese Crosses" in Fabry Disease
}

\author{
Kouichi Utsumi ${ }^{1}$, Futoshi Mitsuhashi², Ken-ichiro Katsura ${ }^{1}$, \\ Yasuhiko Iino ${ }^{1}$ and Yasuo Katayama ${ }^{1}$
}

${ }^{1}$ Department of Neurological, Nephrological and Rheumatological Science, Graduate School of Medicine, Nippon Medical School

${ }^{2}$ The Clinical Laboratory Department, Nippon Medical School

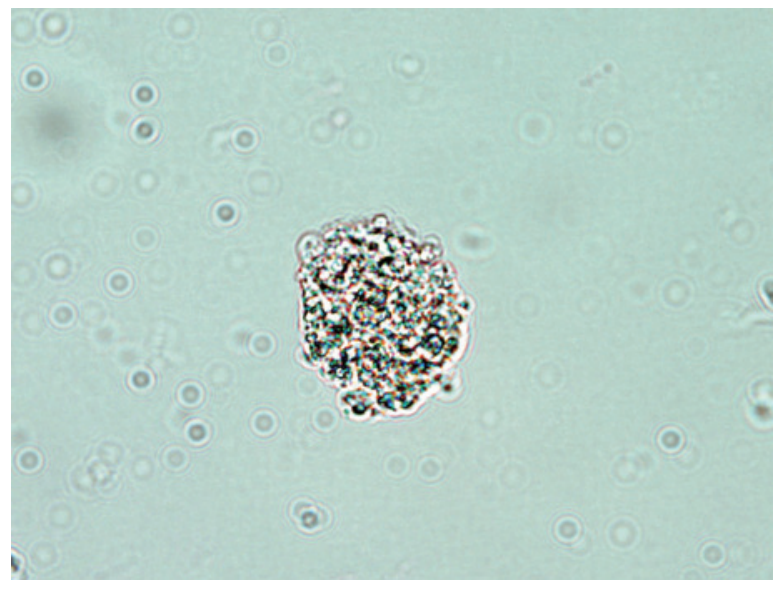

Fig. 1

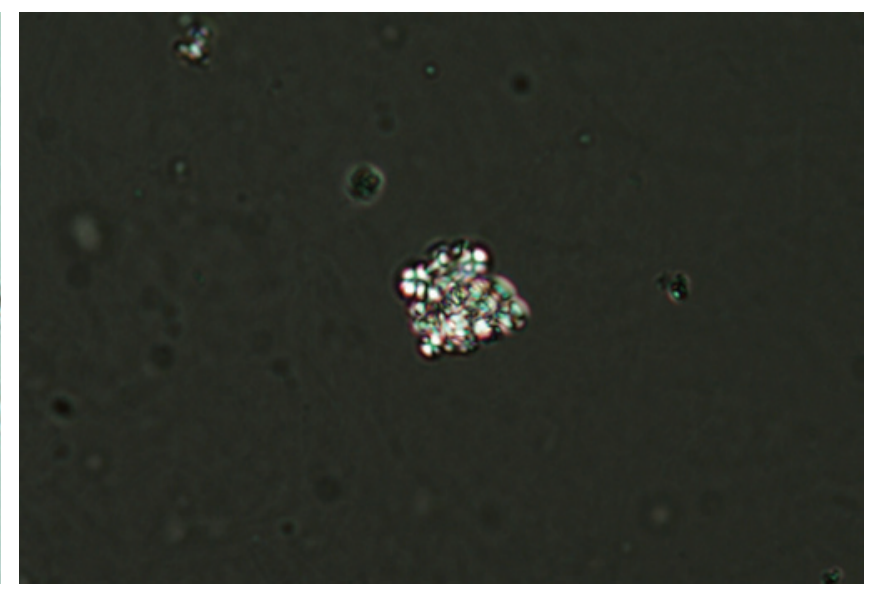

Fig. 2

\begin{abstract}
Fabry disease, an X-chromosome-linked disorder caused by a genetic deficiency of lysosomal $\alpha$-galactosidase $(\alpha$-Gal) A, leads to the systemic accumulation of metabolic intermediates known as glycosphingolipids, particularly globotriaosylceramide (GL 3). Because enzyme replacement therapy is available for Fabry disease, it is important to make an early diagnosis and to start treatment. However, many cases of Fabry disease remain undiagnosed. Here we report on a 41-year-old man with Fabry disease. He had bilateral paresthesia of the calves. Results of laboratory tests were normal, except for the examination of urinary sediment, which showed oval fat bodies (Fig. 1) with typical "Maltese crosses" under polarized light (Fig. 2). No $\alpha$-Gal A activity was detectable activity in the serum or in leukocytes. Accordingly, Fabry disease was diagnosed. The patient had a T-to-A transition at codon 372, resulting in substitution of the predictable glutamine for leucine. Enzyme replacement therapy was started and was repeated every 2 weeks. The appearance of Maltese crosses in the sediment was due to the birefringence of the lipid droplets, which consist mainly of GL 3. Examination of urinary sediment can be done anywhere and is a useful noninvasive screening method for Fabry disease. The first strategy for the treatment of Fabry disease is the detection of undiagnosed cases.
\end{abstract}

Fig. 1 Urinary sediment from this patient showed renal tubular cells as oval fat bodies.

Fig. 2 "Maltese Crosses" in the urinary sediment were detected with polarizing light microscopy.

Correspondence to Kouichi Utsumi, MD, Department of Neurological, Nephrological, and Rheumatological Science, Graduate School of Medicine, Nippon Medical School, 1-1-5 Sendagi, Bunkyo-ku, Tokyo 113-8603, Japan E-mail: utsumi@nms.ac.jpＪournal Website (http://www.nms.ac.jp/jnms/) 